\title{
Relação entre o consumo de açúcares de adição e a adequação da dieta de adolescentes residentes no município de São Paulo ${ }^{1}$
}

\section{$\|$}

Relationship between the intake of added sugars

and dietary adequacy in adolescents from

the municipality of São Paulo, Brazil

Ana Carolina Almada COLUCCI

Chester Luis Galvão CESAR ${ }^{3}$

Dirce Maria Lobo MARCHIONI ${ }^{3}$

Regina Mara FISBERG ${ }^{3}$

\section{R E S U M O}

\section{Objetivo}

Avaliar a relação entre o consumo de açúcares de adição e a adequação do consumo de nutrientes e grupos alimentares em adolescentes residentes no município de São Paulo.

\section{Métodos}

Foram avaliados 793 adolescentes, provenientes de um estudo de base populacional, realizado em 2003. O consumo alimentar foi medido pelo recordatório de 24 horas, tendo sido aplicado método de ajuste por meio de subamostra de 195 indivíduos. O consumo de açúcares foi categorizado em adequado ou inadequado, quando $\leq 10 \%$ ou $>10 \%$ do valor energético total da dieta, respectivamente. A adequação de ingestão de macronutrientes considerou intervalos de distribuição aceitável, e a prevalência de inadequação dos micronutrientes foi calculada pelo método Estimated Average Requirement como ponto de corte. O consumo mediano dos alimentos foi estimado além dos percentis 25 e 75. Foram utilizados testes de Qui-quadrado, Wald e mediana, com nível de significância de $5 \%$.

\footnotetext{
1 Artigo elaborado a partir da tese de A.C.A. COLUCCI, intitulada "Consumo alimentar de açúcares de adição por adolescentes residentes no município de São Paulo". Universidade de São Paulo; 2009. Apoio: Fundação de Amparo à Pesquisa do Estado de São Paulo, processo n 2007/51488-2.

2 Universidade Presbiteriana Mackenzie, Centro de Ciências Biológicas e da Saúde, Curso de Nutrição. São Paulo, SP, Brasil.

3 Universidade de São Paulo, Faculdade de Saúde Pública, Departamento de Nutrição. Av. Dr. Arnaldo, 715, Cerqueira César, 01246-904, São Paulo, SP, Brasil. Correspondência para/Correspondence to: R.M. FISBERG. E-mails: <rfisberg@usp.br>; <regina.fisber@gmail.com>.
} 
220 | A.C.A. COLUCCl et al.

\section{Resultados}

Identificou-se maior proporção de adolescentes com consumo adequado de carboidratos entre aqueles com maior ingestão de açúcares de adição. Todos os adolescentes apresentaram ingestão proteica dentro dos valores preconizados e verificou-se associação significativa entre a adequação de lipídeos e o consumo de açúcares de adição somente entre os adolescentes do sexo masculino. Maior porção mediana de leite, carnes, frutas, suco industrializado, refrigerante e achocolatado em pó foi identificada entre os adolescentes com consumo excessivo de açúcares de adição.

\section{Conclusão}

O consumo excessivo de açúcares de adição se mostrou relacionado à menor adequação do consumo de nutrientes e à menor ingestão de alimentos de alta densidade nutritiva.

Termos de indexação: Açúcar. Adolescente. Consumo de alimentos. Inquéritos sobre dietas.

\section{A B S T R A C T}

\section{Objective}

This study assessed the relationship between intake of added sugars and intake adequacy of nutrients and food groups in adolescents from São Paulo.

\section{Methods}

The study sample comprised 793 adolescents selected from a population-based cross-sectional study conducted in 2003. Food intake was assessed by 24-hour food recalls and an adjustment approach was subsequently administered using a subsample of 195 subjects. Sugar intake was considered appropriate or inappropriate when it represented $\leq 10 \%$ or $>10 \%$ of total energy intake, respectively. Acceptable distribution ranges were used to assess macronutrient intake adequacy. The prevalence of micronutrient inadequacy was estimated using the Estimated Average Requirements as cutoffs. Median food intake and the 25th and 75th percentiles were estimated. The chi-square, Wald and median tests were used to analyze the data. The significance level was set at $5 \%$.

\section{Results}

A larger proportion of adolescents with adequate carbohydrate intake were seen among those with higher added sugar intake. Protein intake by all adolescents was within the recommended ranges. A statistically significant association was found between adequacy of fat intake and added sugar intake among male adolescents. A higher median percent intake of milk, meat, fruit, processed juice, soft drink and chocolate milk was seen among adolescents with excess sugar intake.

\section{Conclusion}

Excessive consumption of added sugars was associated with lower adequacy of nutrient intake and lower intake of nutrient-dense foods.

Indexing terms: Sugar. Adolescent. Food consumption. Diet surveys.

\section{N T R O D U Ç Ã O}

Devido às suas propriedades nutricionais e seus efeitos à saúde humana, o consumo dietético de açúcares de adição tem sido objeto de estudo nas últimas décadas. Tal fato se deve às evidências científicas de que o consumo excessivo de açúcares pode contribuir para o excesso de peso e comprometer a qualidade da dieta pela redução da ingestão de alimentos ricos em nutrientes ${ }^{1,2}$. Apesar de ainda se mostrarem incon- clusivos, alguns estudos têm investigado também a relação entre o consumo de açúcares e a ocorrência de doenças crônicas não transmissíveis, como obesidade, diabetes, câncer, dislipidemias e aterosclerose ${ }^{2}$.

Desde o início dos anos 1960, comitês especializados de diferentes países têm publicado recomendações quanto ao consumo de açúcares. Entre a população norte-americana, a primeira recomendação foi introduzida no ano de 1980 e 
apresentada, de forma simplificada, por meio da mensagem "Evite muito açúcar". Até o ano 2000, a recomendação quanto ao consumo de açúcar restringia-se apenas à orientação de que os indivíduos deveriam escolher bebidas e alimentos de forma a moderar sua ingestão de açúcar, sem menção à quantidade a ser ingerida. Em 2005, foi publicado o Guia Alimentar para a População Americana, que, ao apresentar a mensagem "Escolha e prepare alimentos e bebidas com pouco açúcar de adição, seguindo as quantidades sugeridas na Pirâmide Alimentar", trouxe avanços por utilizar pela primeira vez o termo açúcar de adição e por especificar as quantidades a serem consumidas $^{2}$.

A Organização Mundial de Saúde, em documento publicado em 2003, estabeleceu limites populacionais máximos para o consumo de açúcar em $10 \%$ do consumo energético total ${ }^{1}$, valor também preconizado pelo Guia Alimentar para a População Brasileira, proposto pelo Ministério da Saúde $^{3}$. O guia brasileiro também estabelece o consumo máximo diário de uma porção de alimentos do grupo dos açúcares e doces e indica a redução do consumo de alimentos e bebidas processados com alta concentração de açúcar e das quantidades de açúcares de adição nas preparações caseiras.

Ao longo dos últimos anos, tais iniciativas governamentais para estabelecimento de recomendações nutricionais para o consumo de açúcares têm sido acompanhadas pela realização de pesquisas científicas internacionais que abrangem indivíduos de todas as faixas etárias. Contudo, tendo em vista a importância da qualidade da dieta para o crescimento e o desenvolvimento adequado de indivíduos jovens, grande parte dos estudos existentes é realizada com crianças e adolescentes ${ }^{4-7}$. No Brasil, no entanto, além de serem escassas as pesquisas sobre açúcares de adição, constata-se a inexistência de estudos de base populacional que investiguem a ingestão e a adequação do consumo de açúcares de adição, especialmente por crianças e adolescentes.
Pelo exposto, o presente estudo busca investigar a relação entre o consumo de açúcares de adição e a adequação do consumo de macro e micronutrientes, além de avaliar o consumo de grupos alimentares segundo a adequação da ingestão de açúcares.

\section{MÉ T O D O S}

Trata-se de um estudo transversal, realizado com adolescentes de 12 a 19 anos e 11 meses, de ambos os sexos, por meio de inquérito domiciliar com base populacional, aplicado entre os meses de março e dezembro de 2003.

Para o cálculo do tamanho da amostra, foram definidos grupos de idade e sexo, denominados domínios amostrais. Utilizou-se amostragem estratificada, por conglomerados, em dois estágios. Em cada uma das áreas de pesquisa, os setores censitários foram agrupados em três estratos segundo o percentual de chefes de família com escolaridade de nível universitário. No primeiro estágio da amostragem, foram sorteados, com probabilidade proporcional ao tamanho, dez setores censitários de cada um dos três estratos, o que configurou trinta setores de cada área. No segundo estágio da amostragem, foram sorteados os domicílios. O tamanho mínimo da amostra por domínio foi de 400 indivíduos, valor que possibilitou estimar uma prevalência de 0,5, com erro de amostragem de 0,10, adotando-se um alfa de 0,05 e efeito de delineamento de 2 , considerando-se as possíveis perdas. Após a adoção dos procedimentos de amostragem acima descritos, foram elegíveis para a pesquisa 878 adolescentes. Destes, 33 indivíduos se recusaram a participar $(3,8 \%)$, tendo sido aplicados, portanto, 845 questionários.

Foram excluídos da análise os indivíduos que apresentaram consumo inferior a $500 \mathrm{kcal}$ ou superior a 5 mil kcal $(n=50)$, por serem valores de consumo improváveis, que poderiam sub ou superestimar a ingestão de nutrientes ${ }^{8,9}$, bem como portadores de doenças - diabetes, portadores de tumores malignos ou doenças renais - 
que pudessem interferir no consumo de alimentos ricos em açúcares de adição $(n=2)$. Ao final, a amostra deste estudo foi composta por 793 adolescentes, de ambos os sexos.

A coleta de dados foi realizada por entrevistadores previamente treinados, por meio da aplicação de questionários, sendo o consumo alimentar medido pelo método recordatório de 24 horas. O cálculo do valor nutricional dos alimentos consumidos foi realizado utilizando-se o software Nutrition Data System (NDS) ${ }^{8}$. Quando necessário, o cálculo e a padronização das quantidades e receitas não constantes no programa foram realizados a partir das referências de Pinheiro et al. ${ }^{11,12}$. Nos casos em que ainda restaram dúvidas em relação às receitas, estas foram testadas em Laboratório de Técnica Dietética.

Foram considerados açúcares de adição todos os açúcares adicionados aos alimentos e/ou produtos durante o processamento ou preparo e também o açúcar adicionado ao alimento no momento do consumo (açúcar de mesa). Para obtenção da distribuição do consumo de açúcares de adição, de nutrientes e de alimentos, foram adotados procedimentos estatísticos diferenciados, com o objetivo de obter estimativas mais acuradas do consumo dos adolescentes. Para tais ajustes, foram considerados, além dos dados dos recordatórios coletados no ano de 2003, dados provenientes de uma subamostra de 195 indivíduos, aplicados no ano de 2007.

Assim sendo, para a obtenção da distribuição do consumo de energia e nutrientes, procedeu-se ao ajuste pela variabilidade intra e interpessoal, por meio do método proposto pelo lowa State University (ISU) ${ }^{13}$, com o auxílio do software PC-Side. Para estimativa do consumo dos alimentos pelos adolescentes, adotou-se o método proposto pelo National Cancer Institute ${ }^{14}$, considerado apropriado para obtenção da distribuição do consumo de alimentos. Para a aplicação de tal método, foram utilizadas "macros" (sequências de comandos) disponibilizadas pelo National Cancer Institute ${ }^{15}$, sendo obtida a distribuição por percentil do consumo, em gramas, dos alimentos pelos adolescentes.
O consumo de açúcares de adição foi categorizado, segundo contribuição percentual ao Valor Energético Total da Dieta (VET), em: adequado, quando $\leq 10 \% \mathrm{VET}$, e inadequado, quando $>10 \%$ VET. A adequação de ingestão de macronutrientes em relação ao VET da dieta dos adolescentes considerou os intervalos de distribuição aceitável propostos pelo Institute of Medicine ${ }^{16}$, sendo $45 \%$ a $65 \%$ VET proveniente de carboidratos, $25 \%$ a $35 \%$ de gorduras e $10 \%$ a $30 \%$ de proteínas. Teste de Qui-quadrado ajustado para o desenho amostral foi utilizado para verificar associação entre o sexo e a adequação da ingestão dos macronutrientes e as categorias de consumo de açúcares de adição.

Para as análises deste trabalho, os adolescentes foram divididos em três faixas etárias: 12 a 13 anos, 14 a 18 anos e 19 anos. Tal divisão foi adotada de modo a permitir a comparabilidade dos resultados com os valores estabelecidos nas recomendações nutricionais, que diferem por sexo e faixa etária ${ }^{16}$. Foram calculados a média e o erro padrão de nutrientes ingeridos, e o teste de Wald foi utilizado para verificar a existência de diferenças significativas entre os valores médios de consumo de nutrientes por sexo e por categorias de consumo de açúcares de adição. Teste Quiquadrado foi utilizado para verificar associação entre a adequação da ingestão de macronutrientes (\% VET) e as categorias de consumo de açúcares de adição.

A prevalência de inadequação da ingestão de ferro, zinco, magnésio, fósforo, vitamina $A$, niacina e vitamina $E$ (dados ajustados) foi calculada pelo método Estimated Average Requirement (EAR) como ponto de corte e utilizando-se como padrão de referência os valores de EAR segundo idade e sexo ${ }^{17,18,19,20}$, para categorias com no mínimo 30 indivíduos. O cálculo da prevalência de inadequação foi realizado com o auxílio do software Stata e corresponde à proporção de indivíduos do grupo cujo consumo habitual (estimado previamente por meio da utilização do software PC-Side) esteja abaixo da EAR estabelecida pelo nutriente. Para o cálcio, foi calculada a prevalência 
de indivíduos com consumo acima da Adequate Intake (Al) ${ }^{17}$. Não foi calculada a prevalência de inadequação da ingestão de vitamina $C$, por esse nutriente não ter apresentado aderência à distribuição normal, mesmo após ajuste.

O consumo mediano, em gramas, dos alimentos foi estimado, e foram estabelecidos os percentis 25 e 75 das quantidades consumidas. Utilizou-se o teste da mediana para verificar a existência de diferenças estatísticas das quantidades de alimentos consumidas pelos adolescentes segundo categorias de consumo de açúcares de adição.

As análises de nutrientes consideraram as correções do desenho amostral e foram realizadas no software Stata, módulo Survey (versão 8). As análises de alimentos foram realizadas com o auxílio dos softwares SAS (versão 9.1) e Statistical Package for the Social Sciences (SPSS) (versão 12.0). Na aplicação dos testes estatísticos foram considerados os $p$-valores correspondentes, adotando-se o nível de significância de 5\%. O estudo foi aprovado pelo Comitê de Ética em Pesquisa da Faculdade de Saúde Pública da Universidade de São Paulo, protocolo n 381, em 14 de fevereiro de 2001, e todos os participantes assinaram um termo de consentimento livre e esclarecido antes da inclusão na amostra.

\section{RES ULTADOS}

A amostra deste estudo foi composta por 793 adolescentes, com idade média de 15,56 anos (Erro-Padrão - EP $=0,08$ ), sendo 410 do sexo masculino e 383 do feminino.

Verificou-se que $70 \%$ dos adolescentes do município de São Paulo consomem mais de 10\% do VET provenientes de açúcares de adição, sendo $36 \%$ deles do sexo masculino e $34 \%$ do sexo feminino, sem diferença estatisticamente significativa entre os sexos $(p=0,685)$.

Quanto à ingestão de macronutrientes, em média, carboidratos, proteínas e lipídeos contribuíram, respectivamente, com 51\% (EP=0,17),
$14 \%(E P=0,05)$ e $35 \%(E P=0,13)$ do VET dos adolescentes, não sendo observada diferença significativa entre os sexos. Foi observada ingestão significativamente maior de carboidratos e menor de proteínas e lipídeos entre os adolescentes com consumo de açúcares de adição acima dos valores recomendados.

Identificou-se maior proporção de adolescentes com consumo adequado de carboidratos entre aqueles com maior ingestão de açúcares de adição $(p<0,001)$ e não foi observado consumo de carboidratos acima do recomendado entre os adolescentes do estudo. Quanto ao consumo proteico, todos apresentaram ingestão dentro dos valores preconizados. No que se refere aos lipídeos, foi verificada associação estatisticamente significativa entre a adequação da ingestão desse macronutriente e o consumo de açúcares de adição somente entre os adolescentes do sexo masculino ( $p=0,002)$; aqueles com consumo excessivo de açúcares de adição apresentaram maior adequação de ingestão de lipídeos.

Entre os adolescentes de 12 a 13 anos, foi identificado maior consumo de vitamina $\mathrm{A}$ e de vitamina $C$ pelos adolescentes do sexo masculino com consumo excessivo de açúcares de adição. Para o sexo feminino, ao contrário, foi observada relação inversa entre a ingestão de ferro e niacina e o consumo aumentado de açúcares de adição. Também entre o sexo feminino, foi observada maior proporção de adolescentes com consumo de nutrientes abaixo dos valores recomendados. Para os demais nutrientes, em ambos os sexos, não foi observada diferença significativa de ingestão segundo categorias de consumo de açúcares de adição (Tabela 1).

Entre os adolescentes de 14 a 18 anos, para a maioria dos nutrientes não foi identificada relação com o aumento do consumo de açúcares de adição, com exceção do cálcio (para ambos os sexos) e vitamina A (para os meninos). Observa-se, ainda, que praticamente todos os adolescentes dessa faixa etária não ingerem a quantidade recomendada de vitamina $\mathrm{E}$, o que também ocorre com o magnésio, entre os adolescentes do sexo masculino (Tabela 2). 
224 | A.C.A. COLUCCl et al.

Tabela 1. Média, erro-padrão e adequação do consumo de nutrientes por adolescentes de 12 a 13 anos, segundo o consumo de açúcares de adição, por sexo. São Paulo (SP), 2003.

\begin{tabular}{|c|c|c|c|c|}
\hline \multirow{3}{*}{ Nutriente } & \multicolumn{4}{|c|}{ Consumo de açúcares de adição } \\
\hline & \multicolumn{2}{|c|}{ Masculino } & \multicolumn{2}{|c|}{ Feminino } \\
\hline & Adequado $(n=23)^{3}$ & Inadequado $(\mathrm{n}=71)$ & Adequado $(n=29)^{3}$ & Inadequado $(n=60)$ \\
\hline Cálcio (mg) & 647,72 & 659,80 & 584,50 & 650,39 \\
\hline Erro-padrão & 44,02 & 18,68 & 27,14 & 29,21 \\
\hline Percentual >Al & 4,03 & 0,00 & 0,00 & 1,30 \\
\hline Ferro $(m g)^{\mathbf{b}}$ & 16,62 & 16,42 & 16,39 & 15,85 \\
\hline Erro-padrão & 0,23 & 0,15 & 0,21 & 0,11 \\
\hline Percentual <EAR & - & 0,00 & - & 0,00 \\
\hline Zinco (mg) & 11,29 & 11,14 & 11,11 & 10,94 \\
\hline Erro-padrão & 0,16 & 0,09 & 0,10 & 0,08 \\
\hline Percentual $<$ EAR & - & 0,00 & - & 0,00 \\
\hline Magnésio (mg) & 281,26 & 278,39 & 275,58 & 253,02 \\
\hline Erro-padrão & 9,95 & 6,63 & 12,21 & 5,39 \\
\hline Percentual $<$ EAR & - & 7,48 & - & 10,60 \\
\hline Fósforo (mg) & 1087,25 & 1078,08 & 1043,74 & 1038,78 \\
\hline Erro-padrão & 39,29 & 24,33 & 30,54 & 24,99 \\
\hline Percentual $<$ EAR & - & 45,36 & - & 53,56 \\
\hline Vitamina $A\left(\text { ERA }^{1}\right)^{\mathbf{a}}$ & 553,04 & 604,42 & 596,03 & 590,96 \\
\hline Erro-padrão & 17,38 & 11,40 & 22,69 & 16,90 \\
\hline Percentual <EAR & - & 5,26 & - & 4,93 \\
\hline Niacina $(\mathrm{mg})^{\mathbf{b}}$ & 17,97 & 18,32 & 18,46 & 17,69 \\
\hline Erro-padrão & 0,27 & 0,20 & 0,28 & 0,18 \\
\hline Percentual $<$ EAR & - & 0,00 & - & 0,00 \\
\hline Vitamina E (mg) & 6,14 & 5,99 & 5,83 & 5,43 \\
\hline Erro padrão & 0,33 & 0,18 & 0,24 & 0,15 \\
\hline Percentual $<$ EAR & - & 98,60 & - & 99,97 \\
\hline Vitamina $C^{2}(\mathrm{mg})^{a}$ & 38,79 & 54,47 & 50,62 & 46,74 \\
\hline Erro-padrão & 5,80 & 3,96 & 0,18 & 4,62 \\
\hline Percentual <EAR & - & - & - & - \\
\hline
\end{tabular}

1ERA: equivalentes de atividade do retinol; EAR: estimated average requirement; ${ }^{2}$ não calculada a prevalência de inadequação por não ter distribuição normal; ${ }^{3}$ não calculada a prevalência de inadequação (percentual<EAR) por $n$ ser <30 indivíduos; a diferença significativa $(p<0,05)$ para o sexo masculino; bdiferença significativa $(p<0,05)$ para o sexo feminino.

Quanto aos adolescentes de 19 anos, o consumo excessivo de açúcares leva, entre os do sexo masculino, à maior ingestão de cálcio, e, entre o sexo feminino, à menor ingestão de vitamina E. Os nutrientes que apresentaram grande prevalência de inadequação (maior que 90\%) foram o magnésio e a vitamina $E$ entre o sexo masculino e vitamina E entre o sexo feminino (Tabela 3).

Apesar de apresentar relação direta com a maior contribuição dos açúcares à dieta dos adolescentes, um consumo insuficiente de cálcio (abaixo da Al) foi identificado em aproximadamente $97 \%$ dos adolescentes deste estudo.

Além da análise da relação entre o consumo de açúcares de adição e a ingestão de nutrientes, importa investigar também essa relação com o consumo, em gramas, de diferentes alimentos pelos adolescentes. Foi encontrada maior porção mediana do consumo de leite, carnes, frutas, suco industrializado, refrigerante e achocolatado em pó entre os adolescentes com consumo excessivo de açúcares de adição. O consumo de açúcares de adição em quantidades superiores às reco- 
Tabela 2. Média, erro-padrão e adequação do consumo de nutrientes por adolescentes de 14 a 18 anos, segundo adequação do consumo de açúcares de adição, por sexo. São Paulo (SP), 2003.

\begin{tabular}{|c|c|c|c|c|}
\hline \multirow{3}{*}{ Nutriente } & \multicolumn{4}{|c|}{ Consumo de açúcares de adição } \\
\hline & \multicolumn{2}{|c|}{ Masculino } & \multicolumn{2}{|c|}{ Feminino } \\
\hline & Adequado $(n=77)$ & Inadequado $(n=193)$ & Adequado $(n=69)$ & Inadequado $(n=174)$ \\
\hline Cálcio $(\mathrm{mg})^{\mathrm{ab}}$ & 586,74 & 667,64 & 613,20 & 669,79 \\
\hline Erro-padrão & 20,06 & 13,10 & 20,90 & 15,62 \\
\hline Percentual >Al & 0,97 & 0,00 & 0,00 & 0,46 \\
\hline Ferro (mg) & 16,36 & 16,30 & 16,35 & 16,13 \\
\hline Erro-padrão & 0,10 & 0,07 & 0,13 & 0,08 \\
\hline Percentual $<$ EAR & 0,00 & 0,00 & 0,00 & 0,00 \\
\hline Zinco (mg) & 11,15 & 11,10 & 10,98 & 11,04 \\
\hline Erro-padrão & 0,06 & 0,04 & 0,09 & 0,05 \\
\hline Percentual $<$ EAR & 0,001 & 0,002 & 0,00 & 0,00 \\
\hline Magnésio (mg) & 266,53 & 271,42 & 272,88 & 264,57 \\
\hline Erro-padrão & 4,97 & 3,14 & 6,61 & 4,36 \\
\hline Percentual $<$ EAR & 94,45 & 92,44 & 69,44 & 75,37 \\
\hline Fósforo (mg) & 1029,74 & 1071,67 & 1022,94 & 1071,46 \\
\hline Erro-padrão & 17,54 & 12,19 & 23,37 & 15,87 \\
\hline Percentual $<$ EAR & 56,31 & 46,25 & 56,94 & 46,63 \\
\hline Vitamina A $\left(E^{2} A^{1}\right)^{a}$ & 574,59 & 603,28 & 588,76 & 600,75 \\
\hline Erro-padrão & 8,85 & 6,90 & 8,77 & 7,36 \\
\hline Percentual <EAR & 72,84 & 60,96 & 97,46 & 88,94 \\
\hline Niacina (mg) & 18,11 & 18,35 & 18,08 & 18,20 \\
\hline Erro-padrão & 0,17 & 0,11 & 0,22 & 0,11 \\
\hline Percentual $<$ EAR & 0,002 & 0,00 & 0,00 & 0,00 \\
\hline Vitamina E (mg) & 5,81 & 5,77 & 5,87 & 5,64 \\
\hline Erro padrão & 0,11 & 0,09 & 0,17 & 0,11 \\
\hline Percentual $<$ EAR & 100,00 & 100,00 & 99,90 & 100,00 \\
\hline Vitamina $C^{2}(\mathrm{mg})$ & 51,08 & 57,21 & 58,28 & 55,15 \\
\hline Erro-padrão & 6,78 & 2,70 & 6,54 & 3,09 \\
\hline Percentual $<$ EAR & - & - & - & - \\
\hline
\end{tabular}

'ERA: equivalentes de atividade do retinol; EAR: estimated average requirement; ${ }^{2}$ não calculada a prevalência de inadequação por não ter distribuição normal; a diferença significativa $(p<0,05)$ para o sexo masculino; 'bdiferença significativa $(p<0,05)$ para o sexo feminino.

mendadas não se mostrou relacionado ao tamanho das porções de arroz, feijão, pão francês, legumes e verduras e suco natural consumidas pelos adolescentes (Tabela 4).

\section{I S C U S S Ã O}

A avaliação da influência do consumo de açúcares no consumo de macronutrientes e adequação às recomendações mostram que, mesmo contribuindo para o aumento da ingestão de carboidratos, o excesso do consumo de açúcares de adição não resulta em consumo desse nutriente acima dos valores máximos recomendados. 0 padrão reduzido de consumo de carboidratos aqui observado foi também identificado na população brasileira, de acordo com a Pesquisa de Orçamento Familiar (POF) ${ }^{21}$, que, com base em dados sobre a disponibilidade domiciliar de alimentos, demonstra contribuição percentual de 55,9\% dos carboidratos ao total energético da dieta. Ainda em avaliação da tendência secular do padrão alimentar da população brasileira nos últimos 30 anos, evidencia-se diminuição na participação relativa de carboidratos totais e complexos - asso- 
Tabela 3. Média, erro-padrão e adequação do consumo de nutrientes por adolescentes de 19 anos, segundo o consumo de açúcares de adição, por sexo. São Paulo (SP), 2003

\begin{tabular}{|c|c|c|c|c|}
\hline \multirow{3}{*}{ Nutriente } & \multicolumn{4}{|c|}{ Consumo de açúcares de adição } \\
\hline & \multicolumn{2}{|c|}{ Masculino } & \multicolumn{2}{|c|}{ Feminino } \\
\hline & Adequado $(n=11)^{3}$ & Inadequado ( $\mathrm{n=35}$ ) & Adequado $(n=17)^{3}$ & Inadequado $(n=34)$ \\
\hline Cálcio (mg) $)^{a}$ & 549,84 & 667,60 & 628,74 & 644,96 \\
\hline Erro-padrão & 40,91 & 28,73 & 56,71 & 26,03 \\
\hline Percentual >Al & 0,0 & 2,43 & 3,95 & 2,57 \\
\hline Ferro (mg) & 16,37 & 16,21 & 16,48 & 16,28 \\
\hline Erro-padrão & 0,34 & 0,17 & 0,23 & 0,20 \\
\hline Percentual <EAR & - & 0,0 & - & 0,0 \\
\hline Zinco (mg) & 11,21 & 11,09 & 11,12 & 10,98 \\
\hline Erro-padrão & 0,13 & 0,11 & 0,13 & 0,09 \\
\hline Percentual $<$ EAR & - & 0,47 & - & 0,0 \\
\hline Magnésio (mg) & 271,68 & 266,01 & 280,29 & 273,06 \\
\hline Erro-padrão & 18,81 & 7,64 & 12,24 & 10,59 \\
\hline Percentual $<$ EAR & - & 91,01 & - & 36,76 \\
\hline Fósforo (mg) & 1021,71 & 1072,44 & 1071,74 & 1064,18 \\
\hline Erro-padrão & 51,43 & 27,34 & 52,82 & 27,97 \\
\hline Percentual <EAR & - & 0,06 & - & 0,13 \\
\hline Vitamina A (ERA') & 585,91 & 610,36 & 632,32 & 590,09 \\
\hline Erro-padrão & 39,70 & 19,38 & 18,64 & 12,74 \\
\hline Percentual $<$ EAR & - & 55,74 & - & 17,06 \\
\hline Niacina (mg) & 17,89 & 18,25 & 18,48 & 18,30 \\
\hline Erro-padrão & 0,44 & 0,27 & 0,45 & 0,42 \\
\hline Percentual $<$ EAR & - & 0,0 & - & 0,017 \\
\hline Vitamina $\mathrm{E}(\mathrm{mg})^{\mathbf{b}}$ & 5,88 & 5,58 & 6,18 & 5,51 \\
\hline Erro padrão & 0,49 & 0,21 & 0,30 & 0,19 \\
\hline Percentual $<E A R$ & - & 100,0 & - & 100,0 \\
\hline Vitamina $\mathrm{C}^{2}(\mathrm{mg})$ & 49,10 & 55,55 & 47,28 & 43,36 \\
\hline Erro-padrão & 11,72 & 4,94 & 7,20 & 5,73 \\
\hline Percentual <EAR & - & - & - & - \\
\hline
\end{tabular}

1ERA: equivalentes de atividade do retinol; EAR: estimated average requirement; ${ }^{2}$ não calculada a prevalência de inadequação por não ter distribuição normal; ${ }^{3}$ não calculada a prevalência de inadequação (percentual <EAR) por n ser <30 indivíduos; adiferença significativa ( $\left.p<0,05\right)$ para o sexo

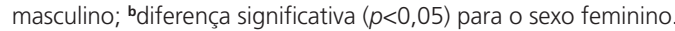

Tabela 4. Porção mediana (P25; P75) dos alimentos consumidos por adolescentes, segundo adequação do consumo de açúcares de adição. São Paulo (SP), 2003.

\begin{tabular}{|c|c|c|c|c|c|c|c|}
\hline \multirow{3}{*}{ Alimento (g) } & \multicolumn{6}{|c|}{ Consumo de açúcares de adição } & \multirow{3}{*}{$p^{*}$} \\
\hline & \multicolumn{3}{|c|}{ Adequado } & \multicolumn{3}{|c|}{ Inadequado } & \\
\hline & Mediana & $\mathrm{P} 25$ & P75 & Mediana & P25 & P75 & \\
\hline Arroz & 226 & 174 & 290 & 216 & 172 & 270 & 0,482 \\
\hline Feijão & 104 & 78 & 136 & 105 & 67 & 147 & 0,888 \\
\hline Leite & 94 & 71 & 123 & 130 & 69 & 190 & 0,003 \\
\hline Carnes & 82 & 68 & 97 & 109 & 83 & 139 & 0,000 \\
\hline Pão francês & 97 & 87 & 110 & 120 & 86 & 120 & 0,325 \\
\hline Frutas & 17 & 5 & 60 & 34 & 23 & 48 & 0,003 \\
\hline Legumes e verduras & 35 & 25 & 36 & 30 & 24 & 38 & 0,326 \\
\hline Suco natural & 93 & 66 & 126 & 85 & 53 & 129 & 0,330 \\
\hline Suco industrializado & 72 & 72 & 95 & 130 & 110 & 152 & 0,000 \\
\hline Refrigerante & 121 & 58 & 228 & 225 & 177 & 280 & 0,000 \\
\hline Achocolatado em pó & 8 & 4 & 16 & 15 & 9 & 24 & 0,000 \\
\hline
\end{tabular}

* teste da mediana. 
ciados ao maior teor de fibras e à maior densidade nutritiva - e constata-se a ocorrência de deslocamento da disponibilidade de carboidratos por gorduras ${ }^{3}$.

Em relação à proteína, todos os adolescentes do município de São Paulo apresentaram consumo adequado. Quanto ao consumo de gorduras, grande percentual de adolescentes apresenta consumo excessivo; somente entre os adolescentes do sexo masculino, observou-se que quanto maior a contribuição dos açúcares de adição à dieta, menor a inadequação do consumo lipídico.

Análises superficiais desses resultados poderiam conduzir à suposição de melhora do padrão alimentar dos adolescentes, pela maior adequação da ingestão de carboidratos e lipídeos decorrente do maior consumo de açúcares. No entanto, deve-se atentar que a maior adequação do consumo de carboidratos ocorre à custa de excesso de açúcares e que cerca de metade dos adolescentes consome uma dieta com alto teor de gorduras. Assim sendo, pode-se afirmar que, em termos de macronutrientes, a alimentação dos adolescentes do município de São Paulo caracteriza-se por padrões alimentares inadequados e de risco à saúde.

No presente estudo, os achados relativos aos micronutrientes indicam diferenças, segundo sexo e faixa etária, na relação entre o consumo excessivo de açúcares e a ingestão de vitaminas e minerais. Em todas as idades, observa-se entre os adolescentes do sexo masculino maior ingestão de micronutrientes - especialmente cálcio e vitamina A - entre aqueles com consumo de açúcares acima dos valores recomendados. Entre os adolescentes do sexo feminino, somente o cálcio apresentou relação direta com a ingestão excessiva de açúcares. Para os demais nutrientes, contudo, o aumento da contribuição dos açúcares de adição à dieta resultou na menor ingestão de micronutrientes, dentre os quais o ferro, a niacina e a vitamina $\mathrm{E}$.

Quase a totalidade dos adolescentes do município de São Paulo consome uma dieta com quantidade insuficiente de vitamina $\mathrm{E}$ (todas as faixas etárias) e vitamina A (acima de 14 anos). 0 consumo de fósforo se mostrou abaixo dos valores recomendados em aproximadamente $50 \%$ dos adolescentes menores de 18 anos e o consumo insuficiente de magnésio foi identificado em cerca de $90 \%$ dos adolescentes do sexo masculino e $45 \%$ do sexo feminino acima de 14 anos.

Segundo Dietary Reference Intakes (DRI), a ingestão diária de fósforo para indivíduos de 9 a 18 anos é de $1055 \mathrm{mg}$, quase o dobro da necessidade estimada para indivíduos acima de 19 anos $(580 \mathrm{mg} / \mathrm{dia})^{17}$. Apesar de a ingestão média dos adolescentes aqui estudados ser de aproximadamente $1000 \mathrm{mg}$ por dia para todas as faixas etárias, diferenças nos valores recomendados, sem dúvida, exercem efeito importante na adequação da ingestão desse nutriente.

Os dados existentes sobre a relação entre o consumo dos açúcares de adição e a qualidade da dieta e a ingestão de micronutrientes ainda se mostram insuficientes para que possam ser estabelecidas evidências conclusivas. Alguns achados apontam efeito negativo do consumo excessivo de açúcares de adição, o que contribui para o excessivo consumo energético e para a redução da densidade nutritiva ${ }^{22,23}$, porém diversos autores observaram associação positiva entre a ingestão de nutrientes essenciais e o aumento da ingestão de açúcares ${ }^{4,24,25}$. Joyce \& Gibney ${ }^{26}$, em estudo recente com crianças e adolescentes irlandeses, observaram redução da ingestão de magnésio, cálcio, zinco e vitaminas $B_{12}$ e $C$ conforme aumento da contribuição percentual dos açúcares de adição ao VET, expresso em tercis de consumo.

Diversos são os estudos que abordam os efeitos do consumo de açúcares de adição sobre a qualidade da dieta, em especial em indivíduos jovens. No entanto, a maioria desses trabalhos apresenta redução ou aumento da ingestão de nutrientes em decorrência do consumo excessivo de açúcares, porém não relaciona essa informação aos valores recomendados de nutrientes para a faixa etária. 
De modo geral, a análise da contribuição dos açúcares de adição à dieta dos indivíduos se dá de duas diferentes formas: tratando-se a contribuição dos açúcares como uma variável contínua ou estabelecendo-se categorias de ingestão que, na maioria das vezes, incluem como ponto de corte os valores máximos de consumo estabelecidos por órgãos de saúde. Tal abordagem possibilita diferenciar os indivíduos com consumo adequado e inadequado e permite construir uma reflexão crítica sobre a aplicabilidade de tais recomendações nutricionais em nossa população. No que diz respeito aos limites estabelecidos, no presente estudo foi utilizado como ponto de corte o limite máximo de $10 \%$ do consumo calórico total proveniente de açúcares, conforme estabelecido pelo Guia Alimentar para a População Brasileira ${ }^{3}$, em consonância com o preconizado pela Organização Mundial de Saúde 1 . Apesar de terem sido adotados para análise da adequação do consumo dos micronutrientes os valores recomendados pelo $10 \mathrm{M}^{17-20}$ - devido à inexistência de recomendações nacionais -, não foi utilizado o limite máximo de $25 \%$ do VET para açúcares de adição, por se considerar tal valor excessivamente elevado.

A análise quantitativa do consumo alimentar envolve, inicialmente, a estimativa dos macro e micronutrientes e, em seguida, a comparação dos resultados obtidos com valores recomendados. No que diz respeito aos macronutrientes, para a população brasileira, essa avaliação pode basear-se nos intervalos de distribuição propostos pelo Guia Alimentar para a População Brasileira ${ }^{3}$. No entanto, apesar de o Ministério da Saúde indicar a utilização de tais diretrizes para todas as fases da vida, tal documento não apresenta valores de contribuição dos macronutrientes específicos para cada faixa etária - como o fazem as recomendações do Institute of Medicine ${ }^{16}$-, o que justifica a utilização das recomendações internacionais na presente pesquisa.

A literatura nacional sobre a avaliação quantitativa da dieta de adolescentes é escassa, e, além disso, diferentes abordagens metodoló- gicas para análise dos dados dietéticos dificultam sensivelmente a comparabilidade dos dados aqui obtidos com os já existentes no país. No presente estudo, foram adotados os valores estabelecidos pelas Dietary Reference Intakes ${ }^{17-20}$ para análise da adequação quantitativa dos micronutrientes ingeridos pelos adolescentes. Morimoto et al. ${ }^{27}$, utilizando análise semelhante em estudo transversal com estudantes do sexo feminino, identificaram inadequação da ingestão de zinco e cobre e, corroborando os resultados aqui apresentados, identificaram aproximadamente $95 \%$ das adolescentes com ingestão de cálcio abaixo da Al.

Também Santos et al. ${ }^{28}$, em pesquisa com estudantes de 10 a 19 anos do município de Ouro Preto (MG), identificaram somente $8 \%$ dos adolescentes com ingestão de cálcio superior à Al. A relação entre a prevalência de inadequação de nutrientes e o consumo de açúcares de adição ainda não foi demonstrada em dados nacionais e poucos são os trabalhos internacionais. Joyce \& Gibney ${ }^{26}$ demonstraram maiores prevalências de inadequação de vitamina A, folato, cálcio, magnésio e zinco entre adolescentes com maior consumo de açúcares. Dados referentes a adolescentes norte-americanos também apontam elevadas prevalências de inadequação de vitamina E e magnésio, mesmo entre aqueles com maior consumo de açúcares ${ }^{16}$.

A análise de consumo de alimentos e sua relação com a ingestão de açúcares são ainda um desafio metodológico decorrente da dificuldade de quantificação do consumo, uma vez que, ao contrário da ingestão diária da maioria dos nutrientes, muitos alimentos e grupos alimentares são consumidos esporadicamente. Em suma, a obtenção da quantidade usualmente consumida de determinados alimentos por um grupo populacional necessita que se considere não somente a quantidade consumida, mas também a probabilidade de consumo desse alimento. Nesse cenário, o presente trabalho apresenta avanços metodológicos para ajuste do consumo de alimentos por meio da utilização de modelos estatísticos que permitiram a obtenção da distribuição percentilar do consumo. 
Maior porção mediana do consumo de leite, carnes, frutas, suco industrializado, refrigerante e achocolatado em pó foi observada entre os adolescentes com consumo excessivo de açúcares de adição. Tais resultados parecem demonstrar que o leite é habitualmente consumido pelos adolescentes com a adição de achocolatado em pó. Porém, apesar de a porção mediana de leite entre os jovens com maior consumo de açúcares ser quase duas vezes maior do que a quantidade ingerida por aqueles com consumo adequado de açúcares, a ingestão diária de cálcio da grande maioria dos adolescentes está muito aquém dos valores recomendados para esse nutriente.

Os resultados aqui obtidos apontam que, para os adolescentes, as ações de intervenção para o aumento do consumo de cálcio devem não somente estimular o aumento da ingestão diária de leite e derivados, mas também enfatizar a necessidade de redução da quantidade de achocolatado adicionado. Sugere-se, ainda, que tais ações estimulem o aumento do consumo de outros alimentos ricos em cálcio, como queijos e iogurtes, por essa população. Apesar de o consumo de açúcares acima das recomendações estar relacionado ao maior consumo de frutas e não ter se mostrado associado à quantidade de legumes e verduras consumidos pelos adolescentes, chama atenção a reduzida quantidade desses alimentos consumida por esse grupo populacional. Alguns estudos científicos têm claramente demonstrado o baixo consumo de hortaliças e frutas $^{28,29}$ e também a pouca frequência e variedade desses alimentos na dieta dos adolescentes ${ }^{30}$.

Configurando-se como os principais contribuintes para a ingestão de açúcares de adição, as porções medianas de consumo de refrigerante e achocolatado em pó entre os adolescentes com consumo excessivo de açúcares são quase duas vezes maiores do que as porções consumidas por aqueles com consumo adequado de açúcares. Enns et al. ${ }^{31}$, ao analisarem as mudanças na composição da dieta de adolescentes norte-americanos de 1977 a 1996, observaram intenso aumento do consumo de alimentos e bebidas ricos em gorduras e açúcares e, ao mesmo tempo, pobres em nutrientes.

Em um estudo longitudinal com duração de 19 meses, Ludwig et al. ${ }^{32}$ observaram associação entre consumo de bebidas adoçadas e obesidade infantil, tendo os refrigerantes contribuído com $8 \%$ do total de energia consumida pelos adolescentes. Pesquisas do Departamento de Agricultura dos Estados Unidos documentaram um aumento do consumo de bebidas gaseificadas pelos adolescentes entre a década de 1970 e 1990, acompanhado pelo declínio do consumo de leite: somente entre os adolescentes do sexo masculino, o consumo de refrigerantes triplicou entre 1977 e 1994.

Quanto às limitações deste estudo, cabe mencionar a possibilidade de modificação dos hábitos alimentares dos adolescentes no período compreendido entre a aplicação do primeiro e do segundo recordatório. No entanto, mesmo com tal limitação, há que se enfatizar a importância da correção dos dados dietéticos, de modo a se obterem informações mais acuradas acerca do consumo alimentar. Outra limitação - o número insuficiente de indivíduos em algumas faixas etárias, quando estratificados por adequação do consumo de açúcares - impossibilitou a análise da prevalência de inadequação dos micronutrientes, o que resultou em limitada amplitude de discussão dos resultados.

Os resultados aqui apresentados demonstram que o padrão alimentar dos adolescentes do município de São Paulo aponta uma dieta com baixa ingestão de carboidratos e excessiva oferta de gorduras, e, ainda, que o consumo excessivo de açúcares de adição resultou em menor ingestão de macro e micronutrientes. O consumo de açúcares em quantidades superiores às recomendadas mostrou-se relacionado à menor ingestão de alimentos de maior densidade nutritiva e ao maior consumo de alimentos ricos em açúcares. Essas evidências apontam a necessidade de intervenção nutricional para redução do consumo de açúcares de adição e adoção de uma alimentação saudável. 
$230 \mid$ A.C.A. COLUCCl et al.

\section{COLABORADORES}

A.C.A COLUCCI participou da análise, da interpretação dos resultados e da elaboração do artigo. C.L.G. CESAR participou do planejamento da pesquisa e da redação final do artigo. D.M.L. MARCHIONI participou da interpretação dos resultados e da redação do artigo. R.M. FISBERG participou da concepção da pesquisa, da interpretação dos resultados e da redação do artigo.

\section{REFERÊ NCIAS}

1. World Health Organization. Diet, nutrition and the prevention of chronic diseases. Geneva: WHO; 2003.

2. United States Department of Agriculture. Dietary guidelines for Americans. $6^{\text {th }}$ ed. Washington (DC): USDA; 2005.

3. Brasil. Ministério da Saúde. Secretaria de Atenção à Saúde. Guia alimentar para a população brasileira: promovendo a alimentação saudável. Brasília: Ministério da Saúde; 2006.

4. Forshee RA, Storey ML. The role of added sugars in the diet quality of children and adolescents. J Am Coll Nutr. 2001; 20(1):32-43.

5. Overby NC, Lillegaard ITL, Johansson L, Andersen LF. High intake of added sugar among Norwegian children and adolescents. Public Health Nutr. 2004; 7(2):285-93. doi:10.1079/PHN2003515.

6. Kranz S, Smicklas-Wright H, Siega-Riz AM, Mitchell D. Adverse effect of high added sugar consumption on diet intake in American preschoolers. J Pediatr. 2005; 146(1):105-11.

7. Rennie KL, Livingstone MBE. Associations between dietary added sugar intake and micronutrient intake: a systematic review. Br J Nutr. 2007; 97(5): 832-41. doi:10.1017/S0007114507617206.

8. Feskanich D, Rockett HRH, Colditz GA. Modifying the healthy eating index to assess diet quality in children and adolescents. J Am Diet Assoc. 2004; 104(9):1375-83. doi:10.1016/j.jada.2004.06.020.

9. Andrade RG, Pereira RA, Sichieri R. Consumo alimentar de adolescentes com e sem sobrepeso do Município do Rio de Janeiro. Cad Saúde Pública. 2003; 19(5):1485-95. doi:10.1590/S0102-311X 2003000500027.

10. Nutrition Coordinating Center. Nutrition data system [software]. Minneapolis: University of de Minnesota; 2007.

11. Pinheiro ABV, Lacerda EMA, Benzecry EH, Gomes MCS, Costa VM. Tabela para avaliação de consumo alimentar em medidas caseiras. $4^{a}$ ed. São Paulo: Atheneu; 2000.

12. Fisberg RM, Villar BS. Manual de receitas e medidas caseiras para cálculo de inquéritos alimentares: manual elaborado para auxiliar o processamento de inquéritos alimentares. São Paulo: Signus; 2002.

13. Nusser SM, Carriquiry AL, Dodd KW, Fuller WA. A semiparametric transformation approach to estimating usual daily intake distributions. Statistical Assoc. 1996; 91(436):1440-9. doi:10.23 07/2291570.

14. Tooze JA, Midthune D, Dodd KW, Freedman LS, Krebs-Smith SM, Subar AF, et al. A new statistical method for estimating the usual intake of episodically consumed foods with application to their distribution. J Am Diet Assoc. 2006; 106(10): 1575-87. doi:10.1016/j.jada.2006.07.003.

15. National Cancer Institute [Internet]. Bethesda: US National Institute of Health [cited 17 Jun 2008]. Avaliable from: <http://riskfactor.cancer.gov/diet/ usualintakes/macros.html>.

16. Institute of Medicine. Dietary reference intakes for energy, carbohydrate, fiber, fat, fatty acids, cholesterol, protein, and amino acids (macronutrients). Washington (DC): National Academy Press; 2005.

17. Institute of Medicine. Dietary reference intakes for calcium, phosphorus, magnesium, vitamin D, and fluoride. Washington (DC): National Academy Press; 1997.

18. Institute of Medicine. Dietary reference intakes for thiamin, riboflavin, niacin, vitamin B6, folate, vitamin $B_{12}$, pantothenic acid, biotin, and choline. Washington (DC): National Academy Press; 1998.

19. Institute of Medicine. Dietary reference intakes for vitamin A, vitamin K, arsenic, boron, chromium, copper, iodine, iron, manganese, molybdenum, nickel, silicon, vanadium, and zinc. Washington (DC): National Academy Press; 2000.

20. Institute of Medicine. Dietary reference intakes for vitamin C, vitamin E, selenium, and carotenoids. Washington (DC): National Academy Press; 2000.

21. Instituto Brasileiro de Geografia e Estatística. Pesquisa de orçamentos familiares 2002-2003: primeiros resultados - Brasil e regiões [on line]. Brasília; 2004 [acesso 24 fev 2006]. Disponível em: <URL: http://www.ibge.gov.br>.

22. Enns CW, Mickle SJ, Goldman JD. Trends in food and nutrient intakes by children in the United States. Fam Econ Nutr Rev. 2002; 14(2):56-68.

23. Alexy U, Sichert-Hellert W, Kersting M. Associations between intake of added sugars and intakes of nutrients and food groups in the diets of German children and adolescents. Br J Nutr. 2003; 90(2): 441-7. doi:10.1079/BJN2003904. 
24. Gibson SA. Consumption and sources of sugars in the diets of British schoolchildren: are high-sugar diets nutritionally inferior? J Hum Nutr Diet. 1993; 6(4):355-71. doi:10.1111/j.1365-277X.1993.tb00 380.x.

25. Gibney M, Sigman-Grant M, Stanton JL Jr, Keast DR. Consumption of sugars. Am J Clin Nutr. 1995; 62(1):178S.

26. Joyce T, Gibney MJ. The impact of added sugar consumption on overall dietary quality in Irish children and teenagers. J Hum Nutr Diet. 2008; 21(5):438-50. doi:10.1111/j.1365-277X.2008.00 895.x.

27. Morimoto JM, Marchioni DML, Fisberg RM. Using dietary reference intake: based methods to estimate prevalence of inadequate nutrient intake among female students in Brazil. J Am Diet Assoc. 2006; 106(5):733-6. doi:10.1016/j.jada.2006.02.005.

28. Santos LC, Martini LA, Freitas SN, Cintra IP. Ingestão de cálcio e indicadores antropométricos entre adolescentes. Rev Nutr. 2007; 20(3):275-83. doi:10.15 90/S1415-52732007000300006.
29. Godoy FC, Andrade SC, Morimoto JM, Carandina L, Goldbaum M, Barros MBA, et al. Índice de qualidade da dieta de adolescentes residentes no distrito do Butantã, município de São Paulo, Brasil. Rev Nutr. 2006; 19(6):663-71. doi:10.1590/\$1415-52 732006000600003.

30. Toral N, Slater B, Cintra IP, Fisberg M. Comportamento alimentar de adolescentes em relação ao consumo de frutas e verduras. Rev Nutr. 2006; 19(3):331-40. doi:10.1590/S1415-527320060 00300004 .

31. Rolim SS, Carlos JV, Bueno MB, Colucci ACA, Andrade SC, Cesar CLG, et al. Tamanho das porções dos principais alimentos consumidos por adolescentes do município de São Paulo. Nutrire. 2007; 32(3):25-36

32. Enns CW, Mickle SJ, Goldman JD. Trends in food and nutrient intakes by adolescents in the United States. Fam Econ Nutr Rev. 2003; 15(2):15-27.

Recebido em: 25/11/2009

Versão final reapresentada em: 5/8/2010

Aprovado em: 3/11/2010 
